Correspondence to: Dr Francisco Gonzalez, Departamento de Fisiologia, Facultad de Medicina Universidad de Santiago, 15705 Santiago de Compostela, Spain.

Accepted for publication 17 May 1995

1 Tansley JO. Cyst of the vitreous. Trans Am Ophthalmol Soc 1899; 8: 507-9.

2 Traboulsi EI, Jeniffer IL, Pyeritz R, Goldber HK, Haller JA. A new syndrome of myelinated nerve fibers, vitreoretinopathy, and skeletal malformations. Arch Ophthalmol 1993; 111 1543-5.

3 Lusky M, Weinberg D, Kremer I. Vitreous cyst combined with bilateral juvenile retinoschisis. F Pediatr Ophthalmol Strabismus 1988; 25: 75-6. RL. Pigmented free-floating vitreous cysts in
Orellana J, O'Malley RE, McPherson AR, Fon two young adults. Ophthalmology 1985; 92: 297-302.

5 Awan KJ. Biomicroscopy and argon laser photocystotomy of free-floating vitreous cysts Ophthalmology 1985; 92: 1710-1.

\section{Bacterial endophthalmitis after anterior chamber paracentesis}

EDITOR,-Bacterial endophthalmitis is one of the most serious complications of ocular surgery. Extended or complicated intraocular surgery is considered to be a risk factor for endophthalmitis. ${ }^{12}$ We report a case of bacterial endophthalmitis following an otherwise uneventful anterior chamber paracentesis.

\section{CASE REPORT}

A 62-year-old woman presented to the emergency department with a 2 hour history of sudden loss of vision in her right eye. Visual acuity was limited to hand movements in her right eye. The lids, external eye and anterior segment were normal with no sign of inflammation. Funduscopy showed a central retinal artery occlusion with a cherry red spot and reduced arterial circulation Intraocular pressure was $22 \mathrm{~mm} \mathrm{Hg}$ in both eyes. The left eye was normal. The patient had a history of arterial hypertension. There was no evidence of arteritis or diabetes.

Massage of the eye did not improve retina circulation. After application of antibiotic drops (Polyspectran; polymyxin, gramicidine, and neomycin) three times in 5 minutes and disinfection of the lids, an anterior chamber paracentesis was performed with a 29 gauge cannula attached to a $1 \mathrm{~m}$ syringe under topical anaesthesia in the operation room under an operation microscope. Approximately $50 \mu \mathrm{l}$ of aqueous humour were aspirated. Thereafter the eyeball was soft, and there was no fistula at the puncture site. Retinal circulation, however remained diminished. The patient received an antibiotic ointment patch (Polyspectran) and oral acetazolamide. After about 6 hours visual acuity and fundus were unchanged; the anterior chamber had $1+$ cells. Twenty four hours after surgery the patient complained of increasing pain and redness of the right eye. Slit-lamp examination revealed corneal oedema, a $1 \mathrm{~mm}$ hypopyon and fibrin in the anterior chamber. Another diagnostic anterior chamber tap was performed. A Gram stained smear showed Gram positive cocci. Antibiotic treatment with intravenous vancomycin and vancomycin eyedrops was initiated. After a further 12 hour period, the anterior chamber inflammation did not improve and B-scan ultrasonography showed anterior vitreous infiltration. A pars plana vitrectomy with application of intraocular vancomycin was performed. Intraoperatively, purulent material was found infiltrating the anterior vitreous, the anterior chamber, and the zonules. The zonules were partially lysed and the dislocated lens was removed. No retinal infiltration was present. Cultures revealed coagulase negative staphylococci sensitive to vancomycin. Systemic antibiotic treatment with intravenous vancomycin was continued. The intraocular inflammation became quiescent, but visual function did not recover.
COMMENT

Anterior chamber paracentesis is a commonly used diagnostic ${ }^{3}$ and therapeutic ${ }^{4}$ procedure. In the present case the paracentesis was performed in an operating theatre under sterile conditions with the same precautions as for al other intraocular operations. The intraoperative course was uneventful. This case was the only endophthalmitis in this operating theatre in a year. The source of the inoculum could not be determined. Possible sources are contamination of the needle or the ocular surface. We have found no other cases of bacterial endophthalmitis following an anterior chamber paracentesis in recent literature. However, the presented case is reminder that even a paracentesis can have serious complications, and should not be considered an essentially harmless procedure. It should only be performed with strict indications.

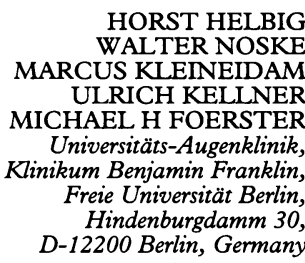

Correspondence to: $\mathrm{H}$ Helbig, Universitäts-

Augenklinik, Klinikum Benjamin Franklin, Freie

Universität Berlin, Hindenburgdamm 30, D-12200 Berlin, German.

Accepted for publication 24 May 1995

1 Aiello LP, Javitt JC, Canner JK. National outcomes of penetrating keratoplasty. Risks of endophthalmitis and retinal detachment. Arch Ophthalmol 1993; 111: 509-13.

2 Menikoff JA, Speaker MG, Marmor M, Raskin EM. A case-control study of risk factors for postoperative endophthalmitis. Ophthalmology postoperative endoph

3 Nussenblatt RB, Palestine AG. Diagnostic testing. In: Uveitis. Fundamentals and clinical practice. Chicago: Year Book Publishers, 1989: 80-93.

4 Augsburger JJ, Magargal LE. Visual prognosis following treatment of acute central retinal artery obstruction. Br $\mathcal{F}$ Ophthalmol 1980; 64: 913-7. 